\title{
Near-Infrared Spectral Geometric Albedos of Charon and Pluto: Constraints on Charon's Surface Composition
}

\author{
TED L. Roush \\ Department of Geosciences, San Francisco State University, 1600 Holloway Avenue, San Francisco, California 941.32; and Space Sciences Division, \\ NASA Ames Research Center, MS 245-3, Moffett Field, California 94035-1000 \\ Dale P. Cruikshank, James B. Pollack, $†$ Ellot F. Young \\ Space Sciences Division, NASA Ames Research Center, MS 245-3, Moffett Field, California 94035-1000 \\ AND \\ MARY J. BARTHOLOMEW \\ Sterling Software, 1121 San Antonio Road, Palo Alto, California 94030 \\ Received August 22, 1994; revised September 25, 1995
}

The spectral geometric albedos of Charon and Pluto are derived at near-infrared wavelengths (1.4-2.5 $\mu \mathrm{m})$ from measurements obtained in 1987. Comparisons of these to theoretical calculations are used to place constraints on the identity and relative abundances of surface ices on Charon. These comparisons suggest that widespread regions of pure $\mathrm{CH}_{4}$ ice do not occur on Charon and that if $\mathrm{CH}_{4}$ is abundant on Charon then it is large grained ( $\approx 5 \mathrm{~mm}$ ) and is likely mixed at the granular level with $\mathrm{H}_{2} \mathrm{O}$ ice, and possibly $\mathrm{CO}_{2}$ ice. 1996 Academic Press, Inc.

\section{INTRODUCTION}

Pluto and its moon Charon may represent two huge icy planetesimals that condensed from the solar nebula in a region where $T<50 \mathrm{~K}$ (Owen et al. 1993) and escaped accretion by the giant planets. They may therefore provide a sampling of the primitive stage in the transition from interstellar materials to the planets and satellites in our Solar System. The Pluto-Charon system also has the highest ratio of the radius of the satellite to the radius of the planet $(\approx 0.5)$, about a factor of 2 greater than that of the Earth-Moon system $(\approx 0.27)$. This may indicate that Pluto and Charon have undergone significantly different internal evolutionary sequences. For example, the Moon is depleted in volatiles relative to the Earth. This is believed to be due to the extensive melting of the lunar surface and interior early in its history (e.g., Warren 1985). Thus, insight into the original composition and subsequent evolu-

\footnotetext{
$\dagger$ Deceased June 13, 1994.
}

tion of Pluto and Charon may be provided by knowledge of their current surface composition.

While Pluto and Charon are in close proximity, they appear to have distinctly different surface compositions. Recent near-infrared (near-IR) spectroscopy of Pluto has revealed that the surface is dominated by $\mathrm{N}_{2}$ ice with minor amounts of $\mathrm{CH}_{4}$ and $\mathrm{CO}$ ices (Owen et al. 1993). Near-IR spectra of Charon have been interpreted as indicating the surface is dominated by $\mathrm{H}_{2} \mathrm{O}$ ice (Buie et al. 1987, Marcialis et al. 1987), although recent theoretical calculations suggest a significant non- $\mathrm{H}_{2} \mathrm{O}$ ice may be present as well (Roush 1994). Roush (1994) found that several calculated spectra of $\mathrm{H}_{2} \mathrm{O}-\mathrm{CO}_{2}, \mathrm{H}_{2} \mathrm{O}-\mathrm{CH}_{4}$, and $\mathrm{H}_{2} \mathrm{O}-\mathrm{CO}_{2}-\mathrm{CH}_{4}$ mixtures could reproduce the shape of the observed Charon spectrum within the errors associated with the Charon data. Comparisons of the calculated spectral geometric albedo $\left(p_{\lambda}\right)$ of these mixtures to the broadband $p_{2.2 \mu \mathrm{m}}$ of Charon (Bosh et al. 1992) eliminated only a few of the mixtures from further consideration (Roush 1994). Greater constraints could be placed on the surface composition if $p_{A}$ were known for Charon at higher spectral resolution over a broader range of near-IR wavelengths.

In this paper we derive near-IR values of $p_{\lambda}$ for Charon from previous observations and provide a comparison to the calculated $p_{\lambda}$ from Roush (1994). In the next section we discuss the telescopic observations, outline our assumptions, and present the values of $p_{\lambda}$ we derive for Charon. In the following section, we compare our derived values of $p_{\lambda}$ to the previous calculations of Roush (1994). Finally, we summarize our results and conclusions. 



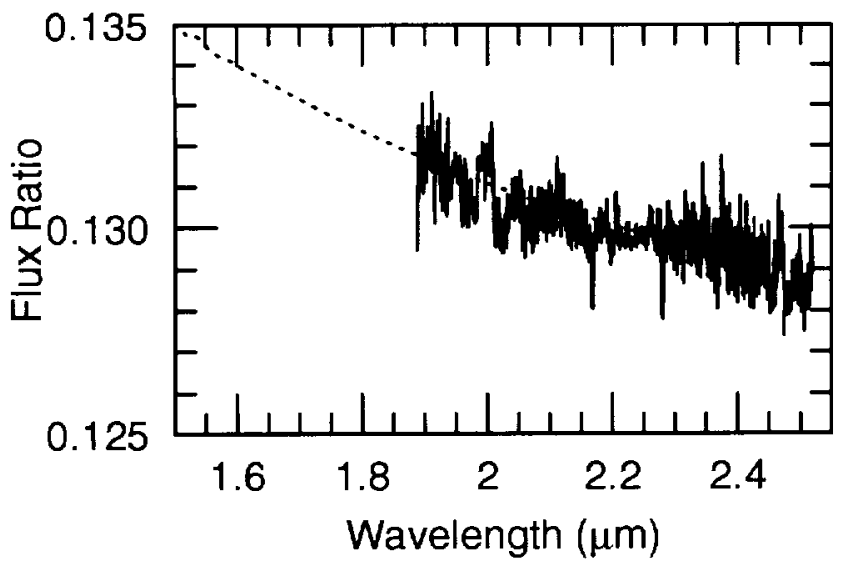

FIG. 1. The flux of SAO120599 relative to BS5384 (solid line) and the flux of a $6030-\mathrm{K}$ blackbody relative to a $5950-\mathrm{K}$ blackbody, scaled to the observational data (dotted line). The blackbody ratio is used to extrapolate the observational data to $1.65 \mu \mathrm{m}$.

\section{OBSERVATIONS AND DERIVATION OF CHARON'S SPECTRAL GEOMETRIC ALBEDO}

Observations. We base our study on the observations of Charon originally obtained by Buie et al. (1987) (referred to hereafter as BEA). The details regarding their acquisition, reduction, and original interpretation are presented in BEA. At the same time BEA also obtained spectral observations of Pluto. For completeness, and to provide another entry in the record of Pluto observations, we derive $p_{\lambda}$ for Pluto below. However, because the focus of this paper is Charon we do not include any discussion or comparisons to the Pluto data. Both of these data were presented as differences in stellar magnitudes, relative to the standard star SAO120599. Digital versions of all the data were kindly provided by Marc Buie (Buie, personal communication, 1993).

Derivation of stellar magnitudes. To derive $p_{\lambda}$ for Pluto and Charon, the near-IR magnitudes of the standard star must be determined. SAO120599 (hereafter referred to as $\mathrm{SAO}$ ) is listed as a G(OV stellar type with a visual magnitude of $8.414 \pm 0.031$ (Turon et al. 1992). A literature search for color properties of this star in the near-IR was unsuccessful. In their derivation of the near-IR fluxes of Pluto and Charon. Marcialis et al. (1987) assumed the near-IR colors of SAO were "solar-like." Because this star is presently close to Pluto in the sky $\left(\alpha=14^{\circ} 41^{\prime} 18.77^{\prime \prime} ; \delta=\right.$ $+03^{\circ} 26^{\prime} 56.5^{\prime \prime} ; \mathrm{J} 2000$ ), it is useful to determine its nearIR spectral energy distribution for future observations. Instead of assuming color properties for SAO, spectral observations of SAO and BS5384 (hereafter referred to as BS) were obtained in May 1995. Figure 1 shows the flux of $\mathrm{SAO}$ relative to $\mathrm{BS}$ as a function of wavelength in the region 1.88 to $2.52 \mu \mathrm{m}$. The $\mathrm{H}$ and $\mathrm{K}$ photometric magnitudes of BS are well documented (Gezari et al. 1993) and after reviewing the various values we adopt $m_{\mathrm{BS} . \mathrm{H}}=$ $4.74 \pm 0.03$ and $m_{\mathrm{BS} . \mathrm{K}}=4.68 \pm 0.03$. The magnitude of the object of interest can be determined via

$$
m_{0}=-2.5 \log _{10}\left(\frac{. \bar{c}}{. \bar{k}}\right)+m_{\mathrm{s}}
$$

where $m_{\mathrm{o}}$ and $m_{\mathrm{s}}$ are the magnitudes of the object and the standard being used, respectively, and the quantity in the brackets is the flux of the object relative to the standard. At $2.2 \mu \mathrm{m}$, the monochromatic wavelength of the $\mathrm{K}$ photometric filter, the value of $\overline{\mathrm{AO}} / \mathrm{BS}$ is $0.130 \pm 0.003$, giving $m_{\mathrm{SAO}, \mathrm{K}}=6.90 \pm 0.03$. Poor seeing conditions and other observational priorities prevented observations of SAO at shorter wavelengths. However, the BEA data extend to wavelengths shorter than those of the observational data, so in order to evaluate these data we extrapolate the data in Fig. 1 to the $\mathrm{H}$ photometric region $(1.65 \mu \mathrm{m})$. We use a ratio of blackbodies representing the effective temperature $\left(T_{\mathrm{c}}\right)$ of SAO and BS. SAO is a G0V stellar type, so we use $T_{\mathrm{c}}=6030 \mathrm{~K}$ (Lang 1991) and BS is a GIV stellar type, so $6030>T_{\mathrm{e}}>5860 \mathrm{~K}$ (Lang 1991). We require the blackbody ratio to reproduce the shape of the observational data. The dotted line in Fig. 1 is a curve correspond-

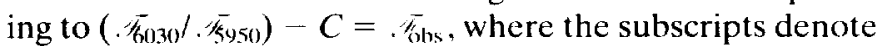
the black body temperature and $C$ is a constant used to scale the flux ratio to the observational data $(C=0.89821)$. The dotted line is used to extrapolate the observed flux ratio to $1.65 \mu \mathrm{m}$ and using this value $(0.134 \pm 0.003)$ yields $m_{\mathrm{SAO}, \mathrm{H}}=6.92 \pm 0.03$. The magnitude of the object (Pluto

TABLE I

Magnitudes and Spectral Geometric Albedos of Charon and Pluto

\begin{tabular}{ccccc}
\hline $\begin{array}{c}\text { Wavelength } \\
(\mu \mathrm{m})\end{array}$ & $m_{\mathrm{C}}$ & $p_{\mathrm{C}}$ & $m_{\mathrm{P}}$ & $p_{\mathrm{P}}$ \\
\hline 1.504 & $14.97 \pm 0.21$ & $0.23 \pm 0.05$ & $12.30 \pm 0.03$ & $0.77 \pm 0.04$ \\
1.595 & $15.00 \pm 0.14$ & $0.22 \pm 0.03$ & $12.72 \pm 0.03$ & $0.52 \pm 0.03$ \\
1.684 & $14.69 \pm 0.10$ & $0.30 \pm 0.03$ & $12.80 \pm 0.03$ & $0.49 \pm 0.03$ \\
1.771 & $14.57 \pm 0.13$ & $0.33 \pm 0.05$ & $12.74 \pm 0.03$ & $0.51 \pm 0.03$ \\
1.858 & $14.62 \pm 0.19$ & $0.30 \pm 0.06$ & $12.42 \pm 0.03$ & $0.65 \pm 0.04$ \\
1.944 & $14.96 \pm 0.17$ & $0.22 \pm 0.04$ & $12.36 \pm 0.03$ & $0.69 \pm 0.04$ \\
2.028 & $15.42 \pm 0.19$ & $0.14 \pm 0.03$ & $12.42 \pm 0.03$ & $0.65 \pm 0.04$ \\
2.111 & $14.97 \pm 0.11$ & $0.22 \pm 0.03$ & $12.74 \pm 0.03$ & $0.48 \pm 0.03$ \\
2.193 & $14.64 \pm 0.09$ & $0.30 \pm 0.03$ & $13.16 \pm 0.04$ & $0.33 \pm 0.02$ \\
2.274 & $14.71 \pm 0.08$ & $0.28 \pm 0.03$ & $13.60 \pm 0.03$ & $0.22 \pm 0.01$ \\
2.353 & $14.89 \pm 0.15$ & $0.24 \pm 0.014$ & $13.58 \pm 0.05$ & $0.22 \pm 0.01$ \\
2.432 & $14.92 \pm 0.22$ & $0.23 \pm 0.05$ & $13.00 \pm 0.04$ & $0.38 \pm 0.02$ \\
2.509 & $14.85 \pm 0.67$ & $0.24 \pm 0.15$ & $12.71 \pm 0.08$ & $0.50 \pm 0.04$
\end{tabular}


or Charon, $m_{0}$ ) is determined via

$$
m_{\mathrm{o}}=\Delta+m_{\mathrm{SAO}},
$$

where $\Delta$ is the magnitude difference as presented in BEA and $m_{\mathrm{SAO}}$ is the magnitude of SAO. We used $m_{\mathrm{SAO.H}}$ for wavelengths $\leq 1.8 \mu \mathrm{m}$ and $m_{\mathrm{SAO}, \mathrm{K}}$ for wavelengths $>1.8$ $\mu \mathrm{m}$. $\mathrm{H}$ and $\mathrm{K}$ solar magnitudes of $-28.188 \pm 0.030$ and $-28.248 \pm 0.030$, respectively, were obtained from Campins et al. (1985). Rearranging Eq. (1). the ratio of the flux from an object to that of the Sun (at 1 AU) can be expressed as

$$
\frac{.5}{.5}=10^{-0.4(m, m)} \text {. }
$$

Derivation of spectral geometric albedos. The spectral geometric albedo is calculated at each wavelength via

$$
\rho_{\lambda}=\frac{R^{2} d^{2}}{r^{2}} \frac{\overline{r_{i}}}{\bar{s}}
$$

where $R$ is the heliocentric distance (in $\mathrm{AU}$ ), $d$ is the geocentric distance (in $\mathrm{AU}$ ), $r$ is the radius of the object (in $\mathrm{AU}$ ), and $/$. in Eq. (4) included radii of $1164 \pm 22.9$ and $621 \pm 20.6$ $\mathrm{km}$ (converted to AU), for Pluto and Charon respectively (Young and Binzel 1994), and 29.6991 and 28.7411 AU for the calculated helio- and geocentric distances on April 23, 1987, when the BEA observations were made at a rotational phase angle $(\phi)$ of 0.75 . At $\phi=0.25$. Charon is between the Earth and Pluto, while at $\phi=0.75$ Pluto is between the Earth and Charon. At $\phi=0.5$ and 1.0 (or $0.0)$ Charon is at southern and northern elongation in its orbit, respectively. The derived magnitudes and spectral geometric albedos are tabulated for each wavelength in Table I, and the spectral geometric albedos are shown graphically in Fig. 2a.

\section{COMPARISONS TO PREVIOUS STUDIES}

Fink and DiSanti (1988) and Marcialis et al. (1992) found that $p_{\lambda} \sim 0.45$ near $1 \mu \mathrm{m}$ for Charon $(\phi=0.75)$. In this paper we find that $p_{\lambda} \approx 0.20 \pm 0.05$ near $1.5 \mu \mathrm{m}$, indicating a significant difference from the $1-\mu \mathrm{m}$ value, although this is not surprising since $\mathrm{H}_{2} \mathrm{O}$ ice has an absorption near 1.5 $\mu \mathrm{m}$. Narrowband filter observations of Charon $(\phi=0.75$, Marcialis et al. 1987) were used to derive values of $p_{\lambda}$ for Charon at four wavelengths assuming a radius of $593 \mathrm{~km}$ (Marcialis et al. 1992). Using a radius of $602 \mathrm{~km}$ for Charon, Bosh et al. (1992) report the $p_{2.2 \mu \mathrm{m}}$ of Charon at rotational phase angles $(\phi)$ of 0.42 and 0.06 . All of these values have been scaled to the Charon radius used here, $621 \mathrm{~km}$, and are compared in Fig. 2b. Inspection of Fig. 2b indicates that the $p_{\lambda}$ derived here are comparable, within the errors, to the values derived by Marcialis et al. (1992) with the notable exception of the $1.5-\mu \mathrm{m}$ value. It is difficult to ascribe the magnitude of this discrepancy to the different assumptions regarding the near-IR colors of SAO. In fact, Marcialis et al. (1987) note some concerns related to the data point at $1.5 \mu \mathrm{m}$. The $2.2-\mu \mathrm{m}$ value derived here is higher than either value presented by Bosh et al. (1992). Convolution of the data presented

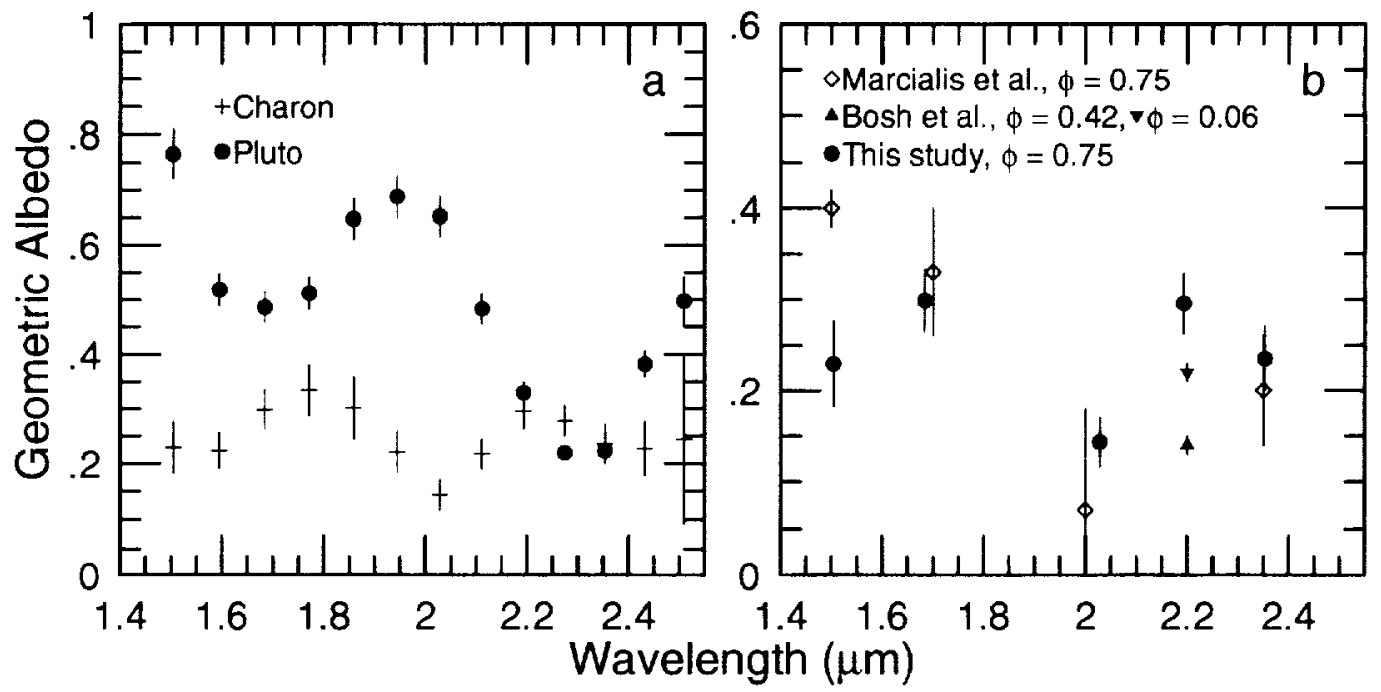

FIG. 2. (a) Spectral geometric albedo of Charon ( + ) using a radius of $62 \pm 20.6 \mathrm{~km}$, and Pluto (O). using a radius of $1164 \pm 22.9 \mathrm{~km}$. Both data sets were derived using the data of Buie ef al. 1987). (b) Comparison of the spectral geometric alhedo values derived here from the Buie et al. (1987) data (O) to values previous reported for (haron: $\Delta$ and $\nabla$ from Bosh et al. (1992): ind $\%$ from Marcialis et al. (1992). 
here, using the nominal $\mathrm{K}$ photometric filter response given in the IRTF photometry manual (Tokunaga 1988), yields a broadband $p_{2.2 \mu \mathrm{m}}$ of $0.23 \pm 0.03$ for $\phi=0.75$ and thus is comparable to the value given by Bosh $e t$ al. (1992) for $\phi=0.06$. Buie and Shriver (1994) present differential magnitudes $\left(m_{\mathrm{C}}-m_{\mathrm{P}}\right)$ for six filter band passes, including the standard $\mathrm{H}$ and $\mathrm{K}$ photometric filters, as a function of Charon's orbital phase, but did not obtain any data at $\phi=0.75$ (at $180^{\circ}$ East Longitude, using their terminology). In the $\mathrm{H}$ filter the differential magnitudes of obtained by Buie and Shriver (1994) range from 1.5 to 2.9 while in the $\mathrm{K}$ filter the range is from 1.56 to 2.4. Convolving the magnitude values in Table I with the nominal IRTF filter responses yields differential magnitudes of $2.17 \pm 0.14$ and $1.90 \pm 0.12$ at $\mathrm{H}$ and $\mathrm{K}$, respectively. These values fall within the range of values defined by the Buie and Shriver data (1994).

\section{COMPOSITIONAL IMPLICATIONS OF CHARON'S SPECTRAL GEOMETRIC ALBEDO}

Roush (1994) calculated the $p_{\lambda}$ using scattering theory for several mixtures of volatile ices representing the surface of Charon. These models included two- and three-component spatial and intimate mixtures consisting of $\mathrm{H}_{2} \mathrm{O}, \mathrm{CO}_{2}$, and $\mathrm{CH}_{4}$ ices. Here we compare $p_{\lambda}$ to some of these mixtures to see if we can further constrain the surface composition of Charon.

A comparison of calculated spatial mixtures to the Charon data is provided in Fig. 3a. The solid line in Fig. $2 \mathrm{a}$ is the best comparison to the Charon data and it corresponds to a spatial mixture of $\mathrm{H}_{2} \mathrm{O}$ and $\mathrm{CO}_{2}$, having grain sizes of 200 and $50 \mu \mathrm{m}$ and relative areal coverages of 80 and $20 \%$, respectively. The other two curves appear to be too bright at the shortest wavelengths, suggesting that the presence of significant occurrences of discrete patches of $\mathrm{CH}_{4}$ ice are unlikely on Charon.

The same comparison for intimate mixtures is presented in Fig. 3b. In this case, two mixtures appear to be comparable to the Charon data, the solid and dashed lines. The solid line corresponds to an intimate mixture of $\mathrm{H}_{2} \mathrm{O}$ and $\mathrm{CO}_{2}$, both having a grain size of $100 \mu \mathrm{m}$ and relative mass fractions of $50 \%$. The dashed line corresponds to a mixture of $\mathrm{H}_{2} \mathrm{O}, \mathrm{CO}_{2}$, and $\mathrm{CH}_{4}$ ices with grain sizes of 100,100 , and $5000 \mu \mathrm{m}$ and relative mass fractions of 35,35 , and $30 \%$, respectively. This suggests that if $\mathrm{CH}_{4}$ ice is present on Charon, then it likely occurs as large particles mixed at the granular level with $\mathrm{H}_{2} \mathrm{O}$, and perhaps $\mathrm{CO}_{2}$, ices.

\section{SUMMARY}

Using the spectral reflectance measurements of Pluto and Charon obtained by Buic et al. (1987), and the radii

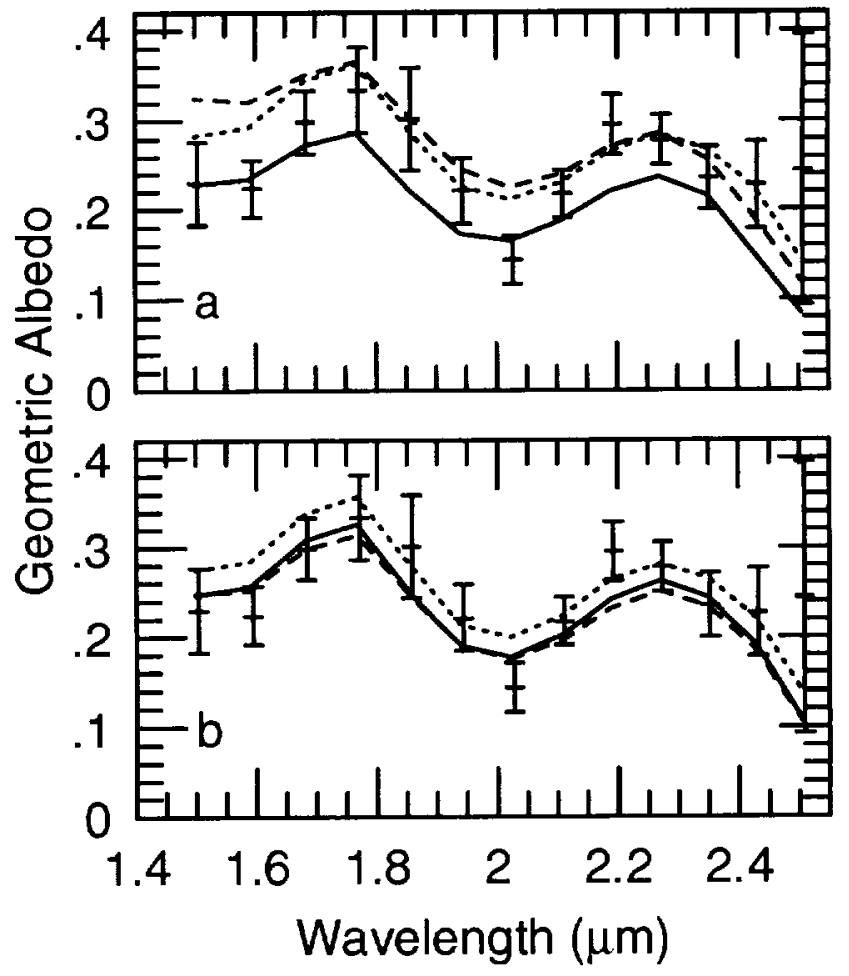

FIG. 3. (a) Comparison of derived spectral geometric albedo of Charon to theoretical calculations of spatial mixtures representing the surface of Charon. The solid line is a mixture of $\mathrm{H}_{2} \mathrm{O}$ and $\mathrm{CO}_{2}$ ices having grain sizes of 200 and $50 \mu \mathrm{m}$, and relative areal coverages of 80 and $20 \%$, respectively. The dotted line is a mixture of $\mathrm{H}_{2} \mathrm{O}$ and $\mathrm{CH}_{4}$ ice having grain sizes of 50 and $25 \mu \mathrm{m}$. and relative areal coverages of 95 and $5 \%$. respectively. The dashed line is a mixture of $\mathrm{H}_{2} \mathrm{O}, \mathrm{CO}_{2}$, and $\mathrm{CH}_{4}$ ice having grain sizes of 100,100 , and $10 \mu \mathrm{m}$ and relative areal coverages of 80,19 , and $1 \%$, respectively. (1) Comparison of derived spectral geometric albedo of Charon to theoretical calculations of intimate mixtures representing the surface of Charon. The solid line is a mixture of $\mathrm{H}_{2} \mathrm{O}$ and $\mathrm{CO}_{2}$ ices each having a grain size of $100 \mu \mathrm{m}$, and relative mass fractions of $50 \%$. The dotted line is a mixture of $\mathrm{H}_{2} \mathrm{O}$ and $\mathrm{CH}_{4}$ ice each having grain sizes of $50 \mu \mathrm{m}$, and relative mass fractions of 95 and $5 \%$, respectively. The dashed line is a mixture of $\mathrm{H}_{2} \mathrm{O}, \mathrm{CO}_{2}$. and $\mathrm{CH}_{4}$ ice having a grain sizes of $1(0), 100$. and 5000$) \mu \mathrm{m}$ and relative mass fractions of 35,35 , and $30 \%$, respectively.

determined by Young and Binzel (1994), we have derived the spectral geometric geometric albedo of these two objects. We find that the values derived here are comparable to values previously reported at similar near-IR wavelengths (Bosh et al. 1992. Marcialis et al. 1992).

Comparison of the derived spectral geometric albedo of Charon to theoretical calculations of spatial mixtures of volatile ices suggest that widespread isolated patches of pure $\mathrm{CH}_{4}$ do not occur on Charon. However, the presence of modest amounts $(\approx 20 \%)$ of isolated patches of $\mathrm{CO}_{2}$ ice remains plausible. Comparisons involving theoretical calculations of intimate mixtures suggest significant 
amounts of $\mathrm{CO}_{2}(50 \mathrm{wt} . \%)$ and $\mathrm{CH}_{4}(30 \mathrm{wt} \%)$ ices may be present, provided the $\mathrm{CH}_{4}$ ice grain size is quite large $(\approx 5 \mathrm{~mm})$. Further progress in determining the surface composition of Charon requires high spectral resolution observations of the satellite spatially resolved from Pluto.

\section{ACKNOWLEDGMENTS}

This research is supported by NASA's Planctary Geology and Geophysics program via RTOP 151-01-60-01. We thank Robert Marcialis, Amanda Bosh. and an anonymous reviewer for their comments.

\section{REFERENCES}

Bosh, A. S., L. A. Young, J. L. Ell.tot, H. B. Hammet, and R. L. Baron 1992. Photometric variability of Charon at $2.2 \mu \mathrm{m}$. Icarus 95, 319-324.

Buie, M. W., D. P. Cruikshank, L. A. Lebofsky. and E. F. Tedesco 1987. Water frost on Charon. Nature 329, 522-523.

Buie, M. W., and S. K. Shriver 1994. The distribution of water frost on Charon. Icarts, 108, 225-233.

CAMPINS, H., G. H. RIEKE, AND M. J. LeBOFSKY 1985. Absolute calibration of photometry at 1 through $5 \mu \mathrm{m}$. Astron. J. 90, 896-899.

Fink, U., And M. A. DiSanti 1988. The separate spectra of Pluto and its satellite Charon. Astron. J. 95, 229-236.

Gezari. D. Y.. M. Schmitz, P. S. PitTs, ani J. M. Mrad 1993. Catalog of Infrared Observations, NASA RP-1294. NASA. Washington, D.C.

LANG, K. R. 1991. Astrophysical Data: Planets and Stars. SpringerVerlag, New York.
Marcialis, R. L., G. H. Rieke, and L. A. Lfbofsky 1987. The surface composition of Charon: Tentative identification of water ice. Science 237, $1349-1351$

Marcialis, R. L., L. A. Libofisky, M. A. DiSanti, U. Fink, E. F. Tedes( (). And J Arrie Ano 1992. The albedos of Pluto and (haron: Wavelength dependence. Astron. J. 103, 1389-1394.

Owen, T. C.. T. L. Rol sh. D. P. Cruikshank, J. L. Eli.iot. I. A. Younci, C. de Berghi. B. So himitit. T. R. Gfbali.e, R. H. Brown, and M. J. BARTHOL OMFW 1993. Surface ices and atmospheric composition of Pluto. Science 261, 745-748.

Roush, T. L. 1994, Charon: More than water ice? lcarus 108, 243-254. Tokunaga, A. T. 1988. The Infrared Telescope Facility Photometry Manual. NASA IRTF, Inst. for Astronomy, Univ. Hawaii, Honolulu, HI.

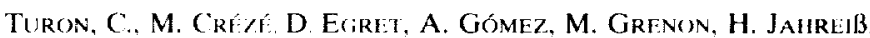
Y. Ŕolutemi, A. N. Argite, A. Ber-Borsenberger, J. Dommangilt, M. O. Mennessifi. F. Arenou, M. Chareton, F. Crifo, J. C. Mermiliód, D. Morin, B. Nicolet, O. Nys, L. Prevot, M. Rolisslial, M. A. C. Perryman, J. E. Ariot, A. Bacilin, D Barthes, M. O. Baylac, P. Brosche, M. Burnet, J. Delhayt. C. Detrbakn, M. Erbach, F. Figiveras, W. Fricke, L. Heimer, P. He.

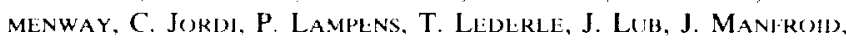
J. A. Mattei, J. M. Maztrier, M. Mermilliold, L. V. Morrison, C. A. Mirray. E. Obi ak. P. P. Périé, B. Pernier, R. S. L.Pomil: L. Quijano, M. Ramaport. A. Sellilik, J. Torra, H.-J. Tuluol kl: ANI C. IF VI:Git 1992. The Hipparcos Input Catalog, ESA SP-1136. ESA, Noordwijk. The Netherlands.

WARREN, P. H. 1985. The magma ocean concept and lunar evolution, Annu. Rev. Earth Planet. Sci, 13, 201-240.

Younci, E. F., AND R. P'. Binztit. 1994. A new determination of radii and limb parameters for Pluto and Charon from mutual event lightcurves. Icarus 108, 219-224. 